\title{
Physics Based Degradation Models for Electrolytic Capacitor Prognostics under Thermal Overstress Conditions
}

\author{
Chetan S. Kulkarni ${ }^{1}$, Gautam Biswas ${ }^{2}$, José R. Celaya ${ }^{3}$, and Kai Goebel ${ }^{4}$ \\ 1,3 SGT Inc., NASA Ames Research Center, Moffett Field, CA, 94035, USA \\ chetan.s.kulkarni@nasa.gov \\ jose.r.celaya@nasa.gov \\ ${ }^{2}$ Vanderbilt University, Nashville, TN, 37235, USA \\ gautam.biswas@vanderbilt.edu \\ ${ }^{4}$ NASA Ames Research Center, Moffett Field, CA, 94035, USA \\ kai.goebel@nasa.gov
}

\begin{abstract}
Electronics subsystems play an increasingly important role in safety critical systems for monitoring, control, and enhanced functionality. Electrolytic capacitors are an important component in ,amy subsystems that range from power supplies on safety critical avionics equipment to power drivers for electro-mechanical actuators. These capacitors are ideal for passing or bypassing low-frequency signals in power supplies but are known to have lower reliability compared to ceramic and tantalum capacitors, and given their criticality in electronics subsystems they are a good candidate for component level monitoring and prognostics. Prognostics provides a way to assess remaining useful life of components and systems based on their current state of health and their anticipated future use and operating conditions. Past experiences have shown that capacitor degradation and failures are quite prevalent under high electrical and thermal stress conditions that they experience during operations. Our focus in this work is on deriving a physics-based degradation model for electrolytic capacitors under thermal stress conditions. As part of our methodology, we study the effects of accelerated aging due to thermal stress on a batch of capacitors stored at high ambient temperature conditions. This provides a framework for supplementing theoretical modeling with data collected from simultaneous experiments, which is then used to validate the derived models. This work represents a first step toward combining data driven and physics-based approaches for modeling capacitor degradation. The case study shows

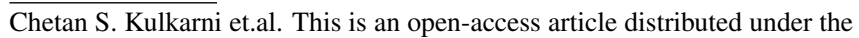
terms of the Creative Commons Attribution 3.0 United States License, which permits unrestricted use, distribution, and reproduction in any medium, provided the original author and source are credited.
}

how the proposed technique is applied to a batch of identically manufactured capacitors.

\section{INTRODUCTION}

Many devices and systems today consist of embedded electronic modules for monitoring, control, and enhanced functionality. However, it has been found that these modules are often the first elements in the system to fail thus reducing overall system reliability (Vichare \& Pecht, 2006; FIDESGroup, 2004). These failures can often be attributed to adverse storage and operating conditions, such as high temperatures, voltage surges and current spikes that the modules and their components may be subjected to during operation. This motivates the need for developing Integrated Vehicle Health Management (IVHM) technologies for systems with embedded electronics. Studying and analyzing the performance degradation of embedded electronics enhances system reliability, assures system performance, avoids catastrophic failures, and reduces maintenance costs over the life of the system (Celaya, Wysocki, et al., 2010; Ferrell, 1999). In addition, an understanding of the deterioration mechanisms helps to systematically track the changes in system behavior and performance, develop the capability to anticipate failures, and predict the remaining useful life (RUL) of the electronic systems long before the components actually fail.

IVHM technologies typically include diagnostics and prognostics functions. The term "diagnostics" relates to the ability to detect and isolate faults or failures in a system. "Prognostics", on the other hand, is the process of predicting the health condition and remaining useful life of components based on current state, and current and future operating conditions. 
Prognostics and health management (PHM) methods combine sensing, data collection, interpretation of environmental, operational, and performance related parameters to compute current system state, infer component health and anticipate damage propagation due to degradation of components. This make them one of the key enablers for predicting system level performance and making decisions on when to perform condition based maintenance, thus providing means for lowering life cycle costs in critical systems. PHM methodologies can be implemented using a variety of techniques that combine trend analysis and parameter estimation methods to model performance degradation as a function of duration of use and history of operating conditions. Systematically developed PHM methods advance the goal of intelligent decision making that in turn allows making better strategic and business based decisions.

In this paper, we develop an effective PHM methodology to enable early detection of failure precursors in a specific type of electrolytic capacitor associated with DC-DC power supplies. Our approach combines physics-based degradation modeling supported by empirical experimental analysis for parameterizing the physics models, and then using these models to predict remaining useful life of electrolytic capacitors as well as their effects on overall system performance. Our hypothesis is that early detection will lead to better tracking of the degradation process, thus enabling better predictions and end of life estimates. As a result, this will result in better overall performance and system reliability. The overall contribution of this paper is the development of a mixed physics-based and data-driven methodology, and demonstration of how it applies to modeling the degradation process and RUL in one type of capacitor. We believe that the methodology is general, and may be extended to structurally similar type of capacitors. We hope to demonstrate that in future work.

\subsection{Motivation}

We examine an example from the aerospace domain, where flight and ground staff need to acquire relevant information that enables accurate estimation of the current health state for all subsystems. This has motivated research projects that focus on accurate detection and isolation of degradation and faults in the system, develop precursors to failure, and predict remaining useful life of the critical components and subsystems of the aircraft after degradation or onset of faults have been detected (Celaya, Saxena, et al., 2010; Balaban et al., 2010). Avionics systems in modern aircraft contain a large number of electronics components in the control, communications and navigation systems. Furthermore, with the introduction of fly-by-wire mechanisms, the number of electronic components in aircraft systems are growing. This proliferation gives rise to the possibility of unknown fault modes, which will affect the ability to detect and diagnose faults in a robust and computationally efficient ways. This increases the importance of understanding how these components degrade, and to use this understanding to anticipate, track, isolate, identify, and predict the remaining useful life of the electronic components (Celaya, Saxena, et al., 2010; Balaban et al., 2010).

A typical avionics system module consists of hardware (power supply, GPS (Global Positioning System) receiver, IMU (Inertial Measurement Unit)) and software (GPS software, INAV - integrated navigation solution) components. Switched-mode DC-DC converter power supplies are widely used in avionics systems because of their high efficiency and compact size. The buck-boost DC-DC converter is used to step up/down voltage depending on the application requirements. Our particular application shown in Figure 1, converts a $28 \mathrm{~V}$ DC voltage from a battery source, to produce the required output voltage of $5 \mathrm{~V}$. The electrolytic capacitors and MOSFET (metal oxide semiconductor field-effect transistor) switches are known to have the highest degradation and failure rates among all of the components in the converter (Goodman, Hofmeister, \& Judkins, 2007). Degraded capacitors have a big impact on the performance and efficiency of the DC-DC converters and also pose the risk of initiating cascading failures in the system.

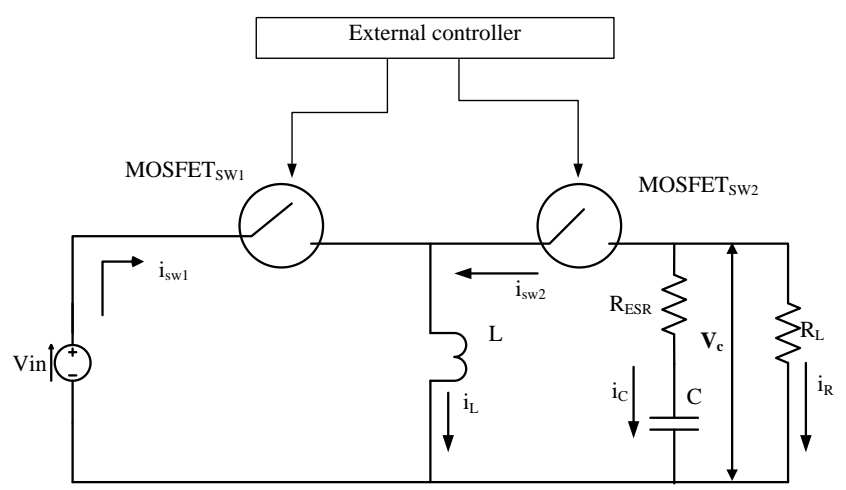

Figure 1. Buck Boost Converter Circuit

Some of the early efforts in diagnostic health monitoring of electronic systems involved the use of a built-in test (BIT), which are on-board hardware-software diagnostic tests to identify and locate faults. Studies conducted by (Pecht et al., 2001) on the use of BITs for fault identification and diagnostics showed a tendency for false alarms, which could result in re-qualification, unnecessary replacements, and loss of system availability, all of which result in increased operation costs.

Degradation in electronic components is harder to detect and analyze as compared to mechanical and structural material, because accurate physics-based models are not readily available for most electronic components (Vichare \& Pecht, 2006). Prognostics failure predictions have to be accurate to support 
decision making for reliability, repair, and replacement. One way to achieve this, is by validating the physics-based degradation models with real data that may be used to refine, verify and validate the accurate time-varying functions that define how parameters change during system operation. Furthermore, component parameters can vary from one manufacturer to another, and between batches manufactured by the same manufacturer (Leonard \& Pecht, 1991). This motivates the need for developing general, first principles approaches for modeling degradation processes and predicting remaining useful life. Data driven methods can supplement the general methodology to derive capacitor degradation models for specific types of capacitors.

\subsection{Research Challenges}

Physics-based degradation modeling considers the primary mechanical, chemical, electrical, and thermal processes that govern the behavior of a component when modeling and the effect of stressors (e.g., higher than rated temperatures, voltage spikes) on component performance. The methodology establishes the cumulative damage due to various failure mechanisms as a function of the state of the component, the relevant physical processes that govern component behavior, and its operating conditions. A failure mode is defined by the causes for the failure (Nasser \& Curtin, 2006). It can also be defined as the way in which a component, subsystem, or system fails to meet or deliver the intended functionality and behaviors. A component can have multiple failure modes, each of which can be derived from the underlying physics-based degradation model associated with the component (Vichare \& Pecht, 2006). In some cases, a theoretical physics of failure model is hard to derive. Researchers have developed empirical methods using selective accelerated life testing to derive the failure mode effects (Nasser \& Curtin, 2006).

Developing failure models from physics-based degradation models is the key to generating accurate prediction models and the remaining useful life of components. However, a number of important challenges remain before these approaches can be applied in real world monitoring, behavior analysis, performance-driven predictions and decision making. The key issues that are currently being addressed in the PHM community include system parameter monitoring, selection of precursors to failures, combining this with parameter monitoring to determine the type of sensors and their placement to ensure tracking of relevant system behavior and operating conditions, in-situ data acquisition, feature extraction for refining the degradation models, and behavior prediction from the collected data (Celaya, Saxena, et al., 2010; Balaban et al., 2010). In addition, it is a challenging task to develop procedures for using the derived physics-based degradation models to predict the overall system performance, and also to understand cascading failures, i.e., how degradation/ failures in components and subsystems affect overall system behavior. In particular, other issues that we have to consider in implementing PHM methodologies for electronic systems include methods for tracking degradation in components, and projecting this degradation to the change in behavior of the subsystems and systems.

\subsection{Organization of Paper}

This paper is organized as follows. Section II discusses current work being done in the area of capacitor fault diagnosis and prognostics. Section III provides a brief overview of physics-based modeling specifically in the electronics and electrical domain and the approach taken in this work. Section IV introduces electrolytic capacitors, their basic structure, operations and degradation mechanisms. Section V discusses our approach and the resulting first principles models for electrolytic capacitor degradation. Section VI presents the thermal overstress experimental setup for accelerated degradation along with validation methods for the derived models. Section VII ends the paper with a discussion, conclusions and directions for future work.

\section{Related Work in CApacitor Diagnosis AND Prognosis}

The high frequency of failure of the output filter capacitor in switched mode power supplies has a critical impact on overall system performance and reliability (Judkins et al., 2007; Vohnout et al., 2008; Goodman et al., 2007; Orsagh \& et'al, 2006). A PHM approach applied to power supplies used in avionics systems is presented in (Orsagh \& et'al, 2006), where aging of the system is attributed to output capacitor and power MOSFET degradation and failures. However, the paper does not discuss the degradation processes or a methodology to derive RUL predictions for the power supply. The work on switched mode power supplies by (Judkins et al., 2007; Goodman et al., 2007) discuss details of the power supply output ripple voltage and leakage current as a function of capacitor degradation, but they do not discuss modeling of the degradation mechanisms and RUL computations in any detail.

A health management approach for multilayer ceramic capacitors is presented in Nie et al. (2007). This approach focuses on a temperature-humidity bias accelerated test to replicate failures, and implements data trending algorithms in conjunction with multivariate data analysis. The Mahalanobis distance method is used to detect abnormalities in the data by using classification methods that label behaviors into "normal" and "abnormal" groups. The abnormal data are then further classified into levels of abnormality by severity based on which predictions are made. In discussions by (Gu et al., 2008), multi-layer ceramic capacitors (MLCC) were selected for in-situ monitoring and life testing under elevated temperature, humidity, and voltage conditions. This method used 
data from accelerated aging tests to detect potential failures and make an estimation of time of failure. A data driven fault detection and prediction algorithm for multilayer ceramic capacitors is presented in ( $\mathrm{Gu} \&$ Pecht, 2008). The prediction approach used in their study combines regression analysis, residual, detection, and prediction analysis. A method based on Mahalanobis distance is used to detect abnormalities in the test data; prediction of RUL is not discussed in this paper.

Wereszczak et al. (1998) discuss the failure probability in Barium Titanate used as a dielectric in MLCC's. Dielectric ceramics in multilayer capacitors are subjected to thermomechanical stresses, which, may cause mechanical failure and lead to loss of electrical function. Probabilistic life design or failure probability analysis of ceramic components combines the strength distribution of the monolithic ceramic material comprising the component, finite element analysis of the component under the mechanical loading conditions of interest, and a multiaxial fracture criterion.

The work by Buiatti et al. (2010) looked at degradation in metalized polypropylene film capacitors, where a noninvasive technique for capacitor diagnostics in boost converters has been discussed. This technique is based on estimations of the ESR (equivalent series resistance) and the capacitance, improving the diagnostic reliability and allowing for predictive maintenance using a low-cost digital signal processor. Imam (2007) discuss a condition based monitoring with implementation of fast fourier transform based prediction approach.

Most of the current work done for capacitor prognostics is based on data driven methodologies. As discussed earlier Nie et al. (2007) implement a trending algorithm based on experimental data, similarly (Gu \& Pecht, 2008) implemented a data driven fault detection and prediction algorithm. In this work we discuss implementation of an hybrid approach, combining data driven and physics based degradation modeling approaches for prognostics.

\section{Physics-based modeling for Prognostics}

In this section we discuss approaches for physics-based degradation modeling in electronics and electrical systems. After discussing the modeling approaches, we present our prognostics approach.

\subsection{Physics-based degradation Modeling}

The physics-based degradation modeling (PBDM) approach to prognosis, models failure mechanisms using a first principles physics-based model of operation, as opposed to traditional approaches that employ data-driven empirical models. These are dynamic models which evolve over time as the device/ system degrades due to usage conditions. Physicsbased degradation modeling techniques are effective for estimating the impact on lifetime due to specific failure mecha- nisms (Hansen \& McCluskey, 2007). Failure predictions, i.e., the time to complete failure from the present time (RUL) and also the evolution of degradation through this time interval, depend upon the current health state of the device/system and the conditions under which it is operated. Therefore, to apply PBDM techniques accurately, one must take into account environmental variables and operating conditions along with a device's physical properties.

In safety-critical complex systems, fault initiation and propagation needs to be determined for accurate and effective fault prognosis. The approach is to derive temporal models of fault propagation and failure using first principles physics-based methods. Recent studies in materials research have focused on microstructural characterization of materials and modeling frameworks that robustly and reliably describe anomaly initiation and propagation as a function of time (Hansen \& McCluskey, 2007). These models, which address the constitutive material properties down to the molecular level, generally are not suitable for real-time treatment due to their computational complexity. Typically, more abstract models, also called lumped models, are usually derived from the microstructural characterizations (Kulkarni et al., 2010). They can be used to estimate and predict degradations in a component/system almost in real time under a given operating environment, such as temperature cycling, vibration, humidity, and electrical stress.

Typically, under normal operating conditions, where parameters are limited to manufacturer-specified threshold limits, a device/ subsystem does not degrade rapidly. In such cases, an experiment may have to be conducted over long periods of time (e.g., months and even years) to detect and study the underlying failure modes. Accelerated stress tests (AST) have been recognized to be a valuable in getting much quicker estimates of the reliability and quality of electronics components (Hansen \& McCluskey, 2007). Accelerated stress tests are often carried out under enhanced adverse environmental conditions to accelerate the damage accumulation rate due to physical or environmental phenomena (Upadhyayula \& Dasgupta, 1998). In traditional accelerated testing techniques, rootcause identification and analysis is not adequately emphasized. In the physics-based degradation modeling (PBDM) approach it is very important to identify, understand, and model the underlying failure mechanisms to derive general (as opposed to situation-specific) models of the aging phenomena.

The main activities in implementing a PHM methodology include:

- using system structure and physical laws to derive a first principles operational model of the device/system;

- identifying failure modes based on stressors (electrical, thermal, mechanical and chemical), which then helps to establish the underlying root cause and derive temporal 
models of component degradation (Watson, 1992);

- deriving relevant degradation/ failure mechanism models

- modifying and enhancing the physics-based models using experimental data and stochastic modeling methods (Hansen \& McCluskey, 2007), and

- using the derived physics based models and prognostic algorithms for predicting the remaining useful life of the system, and

\subsection{Research Approach}

As mentioned earlier, prognostics approaches are essential for improving system safety, reliability, and availability. Prognostics deals with determining the health state of components, and projecting this state into the future to make end of life (EOL) and RUL estimations. Model-based prognostics approaches perform these tasks employing first principles physics models that capture knowledge about the system, its components, and their degradation mechanisms (Daigle \& Goebel, 2011; Saha \& Goebel, 2011). Faults and degradations appear as parameter value changes in the model, and this provides the mechanism for tracking system behavior under degraded conditions (Celaya et al., 2011; Kulkarni et al., 2012).

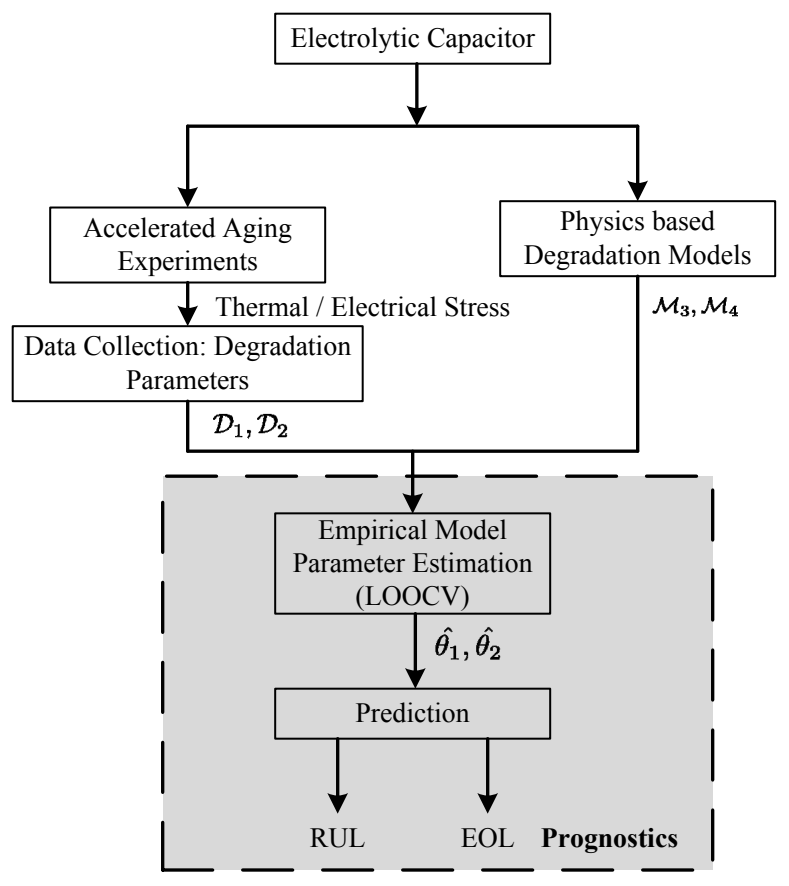

Figure 2. Research Methodology

We implement the prognostics modeling process illustrated in Figure 2. The core of this work focuses on developing physics-based degradation models of components that include descriptions of how fault parameters evolve in time, governed by their operating conditions. As described in Figure 2, we combine experimental studies on the device and the electrical and mechanical configuration information with physics based modeling of behavior phenomena. Identifying the failure precursors and developing accurate models of degradation/ failure from an understanding of the physicsbased model is an important challenge that we address in this research work. Early detection and analysis may lead to more accurate estimation of parameter changes, and therefore, better prediction and end of life estimates of the capacitor. Unknown and time varying parameters in the degradation model are estimated online. Static parameters for the model are estimated using the leave one out cross validation (LOOCV) algorithm. The derived state space models are then implemented in a Bayesian framework for prognostics.

Before discussing the modeling methodology implemented we first discuss in the next section about the structure and type of electrolytic capacitor devices used in this work and degradation mechanisms observed under stress conditions. The study of degradation mechanisms helps in developing and refining the physics based models.

\section{Degradation in Electrolytic Capacitors}

The health of electrolytic capacitors is strongly affected by their operating conditions that include parameters, such as voltage, current, frequency, and ambient temperatures among others. Degradation of capacitors used in DC-DC power converters and signal filters increases the resistive component and decreases the capacitive component of the impedance in the $\mathrm{AC}$ path, which results in an increase in the ripple voltage magnitude that are super imposed on the desired DC voltage. Continued degradation of the capacitor leads the converter output voltage to drop below its specified output value, and this can also affect the health and performance of downstream components. In some cases, this may cause damage to the converter and other downstream components resulting in cascading failures in the systems. We first discuss the internal structure of an electrolytic capacitor and link the different components which lead to degradation in the device.

Figure 3 shows a schematic of the internal components of an capacitor: cathode aluminum foil, electrolytic paper, electrolyte, and an aluminum oxide layer on the anode foil surface, which acts as the dielectric. The electrolyte paper strip is impregnated with liquid electrolyte. When in contact with the electrolyte, the oxide layer provides an excellent forward direction insulation property (Gasperi, 1996). A magnified effective surface area is attained by etching the foil, which attains a high capacitance value in a small volume (Fife, 2006). Since the oxide layer has rectifying properties, a capacitor has polarity. If both the anode and cathode foils are coated with an oxide layer, the capacitors are bipolar (Chen, 2004).

Figure 4 illustrates the unfolded separator paper into which the electrolyte is embedded. The paper height, $h_{c}$ is the height of the capacitor capsule and $l_{c}$ indicates the length of the com- 


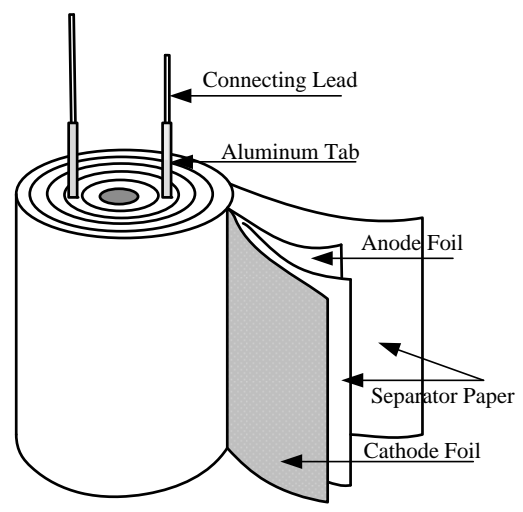

Figure 3. Physical Model of Electrolytic Capacitor

bined roll of paper separator, anode, and cathode. The paper separator is sandwiched between the anode and the cathode. Each has a thickness $d_{S}, d_{A}$ and $d_{C}$, respectively, and can be approximated as $d_{S} \approx d_{A} \approx d_{C}$ for this specific type of capacitor since we are working with low voltage devices.

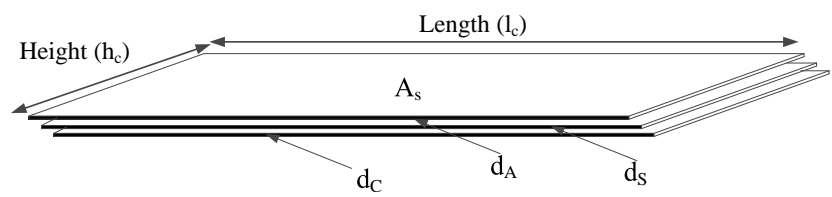

Figure 4. Capacitor open structure

Figure 5 presents a detailed view of the cross section of the electrolytic capacitor structure. The etched anode and cathode plates are anodized by coating them with a thin aluminum oxide layer on the surface of the foil. This layer of aluminum oxide acts as the dielectric (insulator) and serves to block the flow of direct current between anode and cathode foil surface (Fife, 2006). When a DC voltage is applied to a discharged capacitor there is an initial surge of current observed during charging. As the voltage across the terminals of the capacitor increases due to capacitor charging, the current flow drops exponentially, and trends to zero as the capacitor terminal voltage equals the applied voltage (Alwitt \& Parler, 1995).

\subsection{Degradation Mechanisms}

Failures can be one of two types: (1) catastrophic failures, where there is complete loss of functionality primarily short and open circuits, and (2) degradation failures, where there is gradual deterioration of capacitor parameters due to accumulated internal damage. These degradation phenomena cause a drift in the two main electrical parameters of the capacitor: (1) the Equivalent series resistance $(E S R)$, and (2) the capacitance $(C)$. The health of a capacitor is typically measured by the values of these two parameters. Our approach tracks the behavior of the device till complete failure, which manifests as short or open circuits, or opening of the vents.

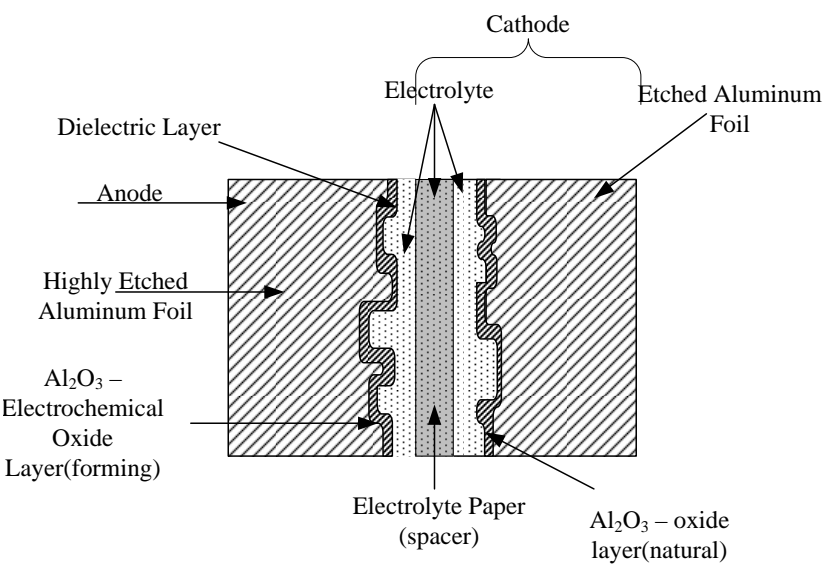

Figure 5. Cross sectional details of internal capacitor structure

The rest of the section describes the degradation mechanisms in terms of the internal structure of the capacitors, and their impact on the electrical parameters of the capacitors.

A study of the literature determines that the primary reasons for degradation in electrolytic capacitors is due to deterioration of electrolyte quality, decreases in electrolyte volume due to evaporation, and weakening of the oxide layer, due to increased temperatures in the capacitor core (Brettle \& Jackson, 1977; Luo, Namburu, Pattipati, \& et'al, 2003). The fishbone diagram in Figure 6 summarizes the comprehensive set of failure modes that occur in electrolytic capacitors (Alwitt \& Parler, 1995; Albella et al., 1984; Wit \& Crevecoeur, 1974; Ikonopisov, 1977; Gomez-Aleixandre et al., 1984; Roederstein, 2007). This diagram identifies the relationship between root causes and failure modes observed in electrolytic capacitors. The major degradation phenomena, represented by the fish bones are:

- Electrolyte Evaporation,

- Degradation of Anode Foil,

- Degradation of Cathode Foil, and

- Degradation/ aging of oxide film.

Elaboration of the main causes, represented as minor bones linking to the degradation/failure causes are:

- Prolonged use,

- Increase in internal temperature,

- Over voltage stress, and

- Charging/discharging cycles.

These are then linked to the finer bones, which explain the root causes for the different failure mechanisms in the capacitor. These root causes can occur individually or in groups depending upon the conditions of operation. 


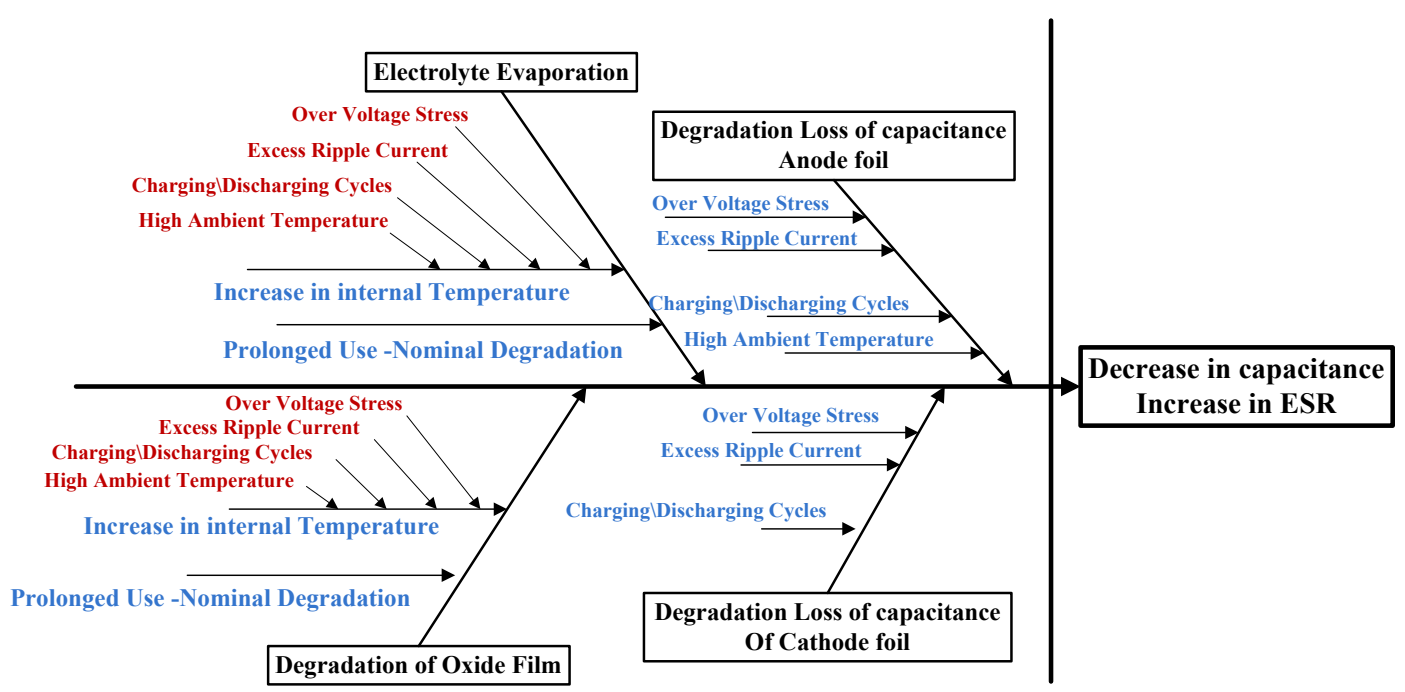

Causes $\backslash$ Failure Mechanisms

Failure Mode

Figure 6. Fishbone diagram of failure mechanisms in aluminum electrolytic capacitor

Our primary focus in this work is to study and model degradation under thermal overstress conditions. Exposure of the capacitors to temperatures that are greater than the rated operating temperatures $\left(T_{\text {rated }}\right)$ results in accelerated aging of the devices (Celaya et al., 2011; IEC-60068-1, 1988). Higher ambient storage temperature accelerates the rate of electrolyte evaporation leading to degradation of the capacitance parameter (Bengt, 1995) prominently than the ESR. Hence we specifically study degradation in capacitance parameter. Referring to the fishbone diagram in Figure 6 thermal stress conditions have a prominent effect on degradation in capacitors due to increase in internal temperature. Models are derived based on the root causes leading to degradation which are studied by conducting experiments and underlying physics.

\section{Capacitor Degradation Models}

In the last section we discussed degradation mechanisms and their link to the physical structure of electrolytic capacitors. Before we take the next step to deriving the physics-based models of the degradation phenomena, we represent the degraded capacitor model as an equivalent lumped parameter electrical circuit. In this section, we first discuss the derived equivalent electrical circuit model of an electrolytic capacitor. We then discuss the link between the equivalent circuit parameters to the physical variables of the capacitor, especially those that are impacted by the degradation process.

\subsection{Equivalent Internal Circuit model for Degraded Ca- pacitor}

A detailed lumped parameter equivalent circuit model of electrolytic capacitors derived from (Morley \& Campbell, 1973) is shown in Figure 7. Inductive parameters are not considered in the model since the capacitors under study are operated in DC mode, therefore, inductive effects can be ignored. The mapping between the electrical circuit components and internal capacitor components are listed below:

1. The resistances $R_{a l}$ and $R_{c l}$ are attributed to the lugs connecting the terminals to the anode and cathode foils, respectively.

2. The elements associated with the anode foil are the resistance of the foil, $R_{a f}$, the capacitance of anode foil, $C_{a f}$, and resistance of oxide in parallel to $C_{a f}, R_{a f p}$ representing the resistive losses caused by the oxide layer.

3. The circuit elements introduced by the electrolyte include the resistance due to the electrolyte in the etched tunnels in the anode and cathode foils, $R_{a o t}$ and $R_{c o t}$, respectively.An additional resistance is due to the electrolyte in the paper separator, $R_{E}$. A parallel capacitance $C_{E}$ represents the dispersion of the paper, which is impregnated with the electrolyte.

4. Similar to the components present in the anode foil, circuit elements associated with the cathode include capacitance of the cathode foil, $C_{c f}$, and resistance of oxide in parallel to $C_{c f}, R_{c f p}$, representing the losses due to the dielectric on the cathode, and finally resistance of the cathode foil, $R_{c o}$.

The cathode capacitance, $C_{c f}$, is typically at least ten times larger in value than the anode capacitance, $C_{a f}$ for low voltage capacitors. This is because the dielectric of the capacitor is present on the anode foil, which is a thin layer of aluminum oxide, $\mathrm{Al}_{2} \mathrm{O}_{3}$ chemically grown on the anode foil using a process know as "formation". During the process of formation 
the effective anode foil surface area is reduced since the microscopic tunnels are partially obstructed by the $\mathrm{Al}_{2} \mathrm{O}_{3}$ deposits, thus reducing the number of tunnels and hence anode capacitance, $C_{a f}$, whereas the formation has no effect on the cathode foil and hence $C_{c f}$ is comparatively very much higher than $C_{a f}$.

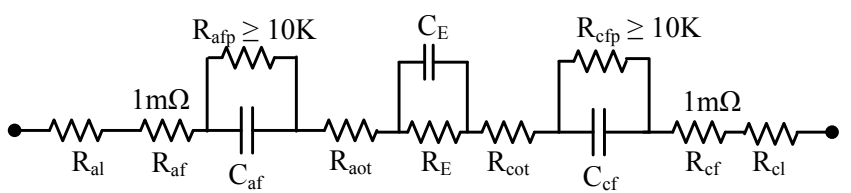

Figure 7. Equivalent electrical model $\left(\mathcal{M}_{1}\right)$ of electrolytic capacitor

This anodization also determines the anode and cathode parallel resistances, $R_{a f p}$ and $R_{c f p}$, respectively. The electrolyte impregnation affects the resistance due to the electrolyte on the separator paper, $R_{E}$ and the parallel capacitance, $C_{E}$ due to dispersion. The set of parameters and their aprroximate expected nominal values (Campbell, 1971) for a typical 2200 $\mu \mathrm{F}$ capacitor are listed in Table 1.

\begin{tabular}{|l|l|}
\hline Parameter & Approximate Value $(\Omega)$ \\
\hline Anode oxide resistance $\left(R_{a f p}\right)$ & $\geq 1 * 10^{4}$ \\
\hline Cathode oxide resistance $\left(R_{c f p}\right)$ & $\geq 1 * 10^{4}$ \\
\hline Series anode foil capacitance $\left(C_{a f}\right)$ & $2400 \mu \mathrm{F}$ \\
\hline Parallel electrolyte Capacitance $\left(C_{E}\right)$ & $\ll C_{a f}$ \\
\hline Series cathode foil capacitance $\left(C_{c f}\right)$ & $\gg C_{a f}$ \\
\hline Resistance of electrolyte in paper $\left(R_{E}\right)$ & $5^{*} 10^{-2}$ \\
\hline $\begin{array}{l}\text { Resistance of electrolyte in etched } \\
\text { tunnels of anode foil }\left(R_{a o t}\right)\end{array}$ & $6^{*} 10^{-3}$ \\
\hline $\begin{array}{l}\text { Resistance of electrolyte in etched } \\
\text { tunnels of cathode foil }\left(R_{c o t}\right)\end{array}$ & $13^{*} 10^{-3}$ \\
\hline Resistance of anode foil $\left(R_{a f}\right)$ & $1 * 10^{-3}$ \\
\hline Resistance of cathode foil $\left(R_{c f}\right)$ & $1 * 10^{-3}$ \\
\hline Lug resistance on anode $\left(R_{a l}\right)$ & $1 * 10^{-4}$ \\
\hline Lug resistance on cathode $\left(R_{c l}\right)$ & $1 * 10^{-4}$ \\
\hline
\end{tabular}

Table 1. Model $\left(\mathcal{M}_{1}\right)$ parameter values associated with capacitor

In this work, our goal is to derive an analytic degradation model of the capacitor, based on the internal capacitor structure and physics-based processes. The parameter values of the model will be derived from experimental data. In order to achieve this, we have to come up with a simpler, abstract form of the model in Figure 8. Our current experimental setup is better suited to directly derive the parameters corresponding to the internal circuit shown in Figure 5. As an approximation, series resistance components $R_{a l}, R_{c l}, R_{a f}, R_{c f}, R_{a o t}$ and $R_{c o t}$ are combined to a single resistance $R_{c}$. Whereas the parallel resistance, $R_{a f p}$ and $R_{c f p}$, each $\geq 1 * 10^{4} \Omega$ in magnitude can be ignored compared to the series resistances that are of the order of $1 * 10^{-3} \Omega$. The cathode foil capac- itance $C_{c f}$ is usually very much greater than the anode foil capacitance, $C_{a f}$, hence $C_{c f}$ can be ignored. The parallel capacitance $C_{E}$ and anode foil capacitance can be combined to form a series capacitance, $C_{c}$.

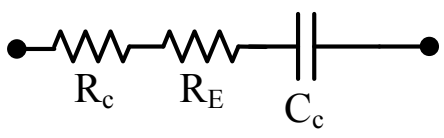

Figure 8. Simplified lumped parameter electrical model $\left(\mathcal{M}_{2}\right)$

As explained from lumped parameter model $\left(\mathcal{M}_{2}\right) E S R$ is approximated as the sum of two component resistances :

$$
E S R=R_{c}+R_{E}
$$

$R_{c}$, as discussed is the combined series resistance of the lugs, cathode and anode foils respectively, which remains almost constant since its a combination of the resistances attributed to mechanical aspects of the capacitor that do not change much over the life of the capacitor. However, as discussed earlier (also, see Table 1) electrolyte resistance, $R_{E}$ is orders of magnitude higher than $R_{c}$, therefore, changes in ESR are primarily influenced by changes in $R_{E}$. (Rusdi et al., 2005) discuss that $R_{c}$ remain relatively constant and $R_{E}$ increases as the capacitor degrades, which can be attributed to the evaporation (i.e.,decrease) of the electrolyte. $R_{c}$ and $R_{E}$ are combined to define the overall degradation resistance, $E S R$ as summarized in Figure 9.

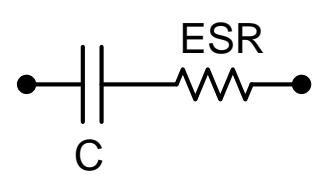

Figure 9. Lumped parameter electrical $\operatorname{Model}\left(\mathcal{M}_{3}\right)$

\subsection{Physics Based Models for $C$ and $E S R$}

The fishbone diagram in Figure 6 discussed a fairly complete set of underlying phenomena that cause capacitor degradation and eventual breakdown. Given our approximate internal lumped parameter electrical circuit model of the capacitor, we have determined that the capacitance value, $C$ and the resistance value, ESR are the primary determinants of capacitor health. We implement a physics modeling approach that links degradation of $C$ and $E S R$ parameters of the capacitor to physical phenomena associated with electrolyte evaporation and oxide layer deterioration. In this paper, with the focus on the effect of thermal stressors on a capacitor under storage conditions i.e., no charge applied. 


\subsubsection{Capacitance Model}

The input impedance of the capacitor network is defined in terms of the total lumped series and parallel impedance of the simplified circuit model (Tasca, 1981) shown in Figure 9. Capacitance represents the ability of a body to store electrical charge. It can be calculated from knowledge of the configuration and the geometry of the conductors and dielectric properties of the insulator between the conductors. Based on the cylindrical geometry the total lumped capacitance for a foil type electrolytic capacitor (Tasca, 1981) is given by:

$$
C=\frac{2 \epsilon_{R} \epsilon_{0} A_{s}}{d_{C}}
$$

where:

$\epsilon_{R}:$ relative dielectric constant,

$\epsilon_{O}:$ permitivity of free space,

$d_{C}$ : oxide layer thickness,

$A_{s}:$ effective oxide surface area.

We hypothesize that the capacitor degrades as the effective oxide area, $A_{s}$ decreases due to evaporation of the electrolyte. The rest of the parameters in Eq. (2) remain relatively unchanged and assumed to be constant over the capacitor lifetime since constant thermal stress is applied and no other stress conditions are considered.

\subsubsection{ESR Model}

The ESR dissipates some of the stored energy in the capacitor. An ideal capacitor would offer no resistance to the flow of current at its leads. Based on the first principles $R_{E}$ is computed as a function of the effective oxide surface area, $A_{s}$ (Tasca, 1981) as:

$$
R_{E}=\frac{1}{2}\left(\frac{\rho_{E} d_{C} P_{E}}{A_{s}}\right)
$$

where:

$\rho_{E}$ : electrolyte resistivity

$P_{E}$ : correlation factor related to electrolyte spacer porosity and average electrolyte pathway.

Since $R_{E} \approx E S R$, increase in $R_{E}$ due to evaporation of electrolyte, which decreases $A_{s}$, will lead to increase in $E S R$, given by :

$$
E S R=\frac{1}{2}\left(\frac{\rho_{E} d_{C} P_{E}}{A_{s}}\right)
$$

Decrease in the electrolyte volume due to thermal overstress, also reduces the average electrolyte path length, which increases $P_{E}$. Under normal circumstances when the capacitors are stored at room temperature or below rated temperatures, no damage or decrease in the life expectancy is observed. But in cases where the capacitors are stored under high temperature conditions the capacitors show irreversible degradation over time. In the next section, we compute the initial electrolyte volume, $V_{e 0}$ using capacitor geometry, and link the oxide surface area, $A_{s}$ parameter to change in electrolyte volume under thermal stress conditions.

\subsection{Computing Electrolyte Volume from Capacitor Ge- ometry}

In section (4.1.) we discussed that under high thermal stress the capacitor electrolyte evaporates at faster rates due to the higher internal temperature. The exact amount of electrolyte in the capacitor is unknown, but we can compute the initial volume of the electrolyte from the dimensions and type of the capacitor. The equation for computing the approximate electrolyte volume is derived from total capacitor capsule volume, $V_{c}$ given by :

$$
V_{c}=\pi r_{c}^{2} h_{c}
$$

where:

$r_{c}:$ radius of capacitor

$h_{c}:$ height of capacitor

From the structural geometry and data in Figure 4, the approximate total internal volume $\left(V_{i}\right)$, neglecting the air gaps created if any in the rolled lumped structure, can be computed as:

$$
V_{i}=A_{s}\left(d_{A}+d_{S}+d_{C}\right)
$$

where:

$d_{A}$ : anode oxide layer thickness,

$d_{C}:$ cathode oxide layer thickness

$d_{S}:$ spacer paper thickness

The total volume :

$$
V_{c}=V_{i}+V_{c a n}
$$

where:

$V_{c}:$ total capacitor volume

$V_{\text {can }}:$ volume of can material

$V_{i}:$ internal volume of can

Referring to the open structure diagram of capacitor shown in Figure 4 we can assume negligible can thickness, $V_{\text {can }} \approx$ 0 , and $d_{A}, d_{S}$ and $d_{C}$ are known from capacitor geometry. Therefore,

$$
\pi r_{c}^{2} h_{c}=A_{s}\left(d_{A}+d_{S}+d_{C}\right)
$$

Correspondingly, the paper spacer volume is given by:

$$
V_{s}=A_{s} d_{S}
$$

The electrolyte is completely soaked in the paper spacer. Hence the total spacer volume can be approximated to be equal to :

$$
V_{s} \approx V_{e 0}
$$


from Eq. (7) and Eq. (8), Eq. ( 6) can be expressed as:

$$
\begin{gathered}
V_{i}=A_{s}\left(d_{A}+d_{C}\right)+A_{s} d_{S} \\
A_{s} d_{S}=V_{s} \\
V_{i}=A_{s}\left(d_{A}+d_{C}\right)+V_{s}
\end{gathered}
$$

Referring to Eq. (9) we get :

$$
V_{i}=A_{s}\left(d_{A}+d_{C}\right)+V_{e 0}
$$

Based on the geometric structure the approximate volume of electrolyte, $\left(V_{e 0}\right)$ in a capacitor can be expressed as follows :

$$
V_{e 0}=V_{i}-A_{s}\left(d_{A}+d_{C}\right)
$$

Referring to Eq. (7) and assumption $V_{\text {can }} \approx 0$ we have,

$$
V_{i}=V_{c}
$$

hence :

$$
V_{e 0}=V_{c}-A_{s}\left(d_{A}+d_{C}\right)
$$

From Eq. (8), Eq. (10) the approximate pristine condition volume of electrolyte, $V_{e 0}$ based on geometry of the capacitor is expressed in terms of following equation:

$$
V_{e 0}=\pi r_{c}^{2} h_{c}-A_{s}\left(d_{A}+d_{C}\right)
$$

Note that Eq.(11) gives us the initial electrolyte volume, $V_{e 0}$, for a specific type of capacitor based on its geometry. The equations need to be updated for a different geometry of capacitors depending upon the number of oxide layers, thickness of oxide layer, type of separator paper used etc.

\subsection{Electrolyte evaporation model}

In last section we used the geometry of the capacitor configuration to estimate the initial electrolyte volume as a function of the capacitor dimensions, and parameter, $A_{s}$, the effective oxide surface area. In this section, we derive the change in electrolyte volume over time caused by evaporation due to thermal stress effects. Therefore, this analysis covers only a small segment of the fishbone diagram, but, nevertheless, is one of the primary contributors to the degradation of electrolytic capacitors.

The evaporation rate, $j_{e o}$ is directly linked with change in the core temperature. The relationship between electrolyte evaporation rate and temperature for a particular electrolyte can be found in (Rusdi et al., 2005). The specific capacitor electrolyte under study in this work consists prominently of ethyl glycol as solvent along with trialkylamines and carboxylic acid. The calculations for evaporation rate are done based on the evaporation of ethyl glycol since it forms the major percentage of the total electrolyte volume. These calculations are to be updated if a different type of electrolyte is used in the capacitor.

When the surrounding temperature of the capacitor capsule is high, heat transfer from the capsule to the capacitor core causes the internal temperature of the capacitor to increase, and as a result the electrolyte evaporates at a faster rate. Under prolonged high temperate exposure the electrolyte evaporation accelerates, which in turn decreases, $A_{s}$ leading to decreases in $C$ (Rodner, Wedin, \& Bergstram, 2002; Rusdi et al., 2005; Bengt, 1995), which is given by :

$$
V_{e}(t)=V_{e 0}-\left(A_{s} j_{e o} w_{e}\right) \times t
$$

where:

$V_{e}$ : dispersion volume at time $\mathrm{t}$

$V_{e 0}$ : initial electrolyte volume

$w_{e}$ : volume of ethyl glycol molecules

$j_{e o}:$ evaporation rate $\left(h r^{-1} \mathrm{~cm}^{-2}\right)$

$t:$ aging time in hours.

From Eq.(12) we derive:

$$
A_{s}=\frac{V_{e 0}-V_{e}(t)}{j_{e o} w_{e} t}
$$

\subsection{Time dependent degradation Models}

Using the physics-based model of electrolyte evaporation, we derive the time dependent degradation models for capacitance $(C)$ and ESR. Using Eq. (2) and Eq. (13) we derive the $c a$ pacitance degradation model as :

$$
\mathcal{D}_{1}: C(t)=\left(\frac{2 \epsilon_{R} \epsilon_{0}}{d_{C}}\right)\left(\frac{V_{e 0}-V_{e}(t)}{j_{e o} t w_{e}}\right),
$$

where $V_{e 0}$ is computed from Eq. (11) and the other parameters have been defined specifically for each capacitor depending upon its structure and manufacturing specifications. Evaporation rate, $j_{e o}$ is a temperature dependent parameter, while electrolyte volume changes with time and evaporation rate. Similarly increase in $E S R$ is given by the increase in electrolyte resistance $\left(R_{E}\right)$, is computed from Eq. (3) and Eq. (12) as :

$$
\mathcal{D}_{2}: \operatorname{ESR}(t)=\frac{1}{2}\left(\rho_{E} d_{C} P_{E}\right)\left(\frac{j_{e o} t w_{e}}{V_{e 0}-V_{e}(t)}\right)
$$

In model, $\mathcal{D}_{2}$ the parameters which are temperature dependent are the rate of evaporation $j_{e o}$ and the correlation factor, $P_{E}$ related to electrolyte spacer porosity and average electrolyte pathway. As the electrolyte evaporates due to high temperature the correlation factor $P_{E}$ will increase as the average pathway of the liquid decreases. Electrolyte evaporation under thermal stress storage conditions can be attributed 
to increase in the temperature of the capacitor capsule. Heat travels from the surface of the body to the core and this phenomenon is described by a thermal model in (Kulkarni et al., 2009, 2011).

\section{Thermal OVERSTRESS EXPERIMENTS}

We setup an experimental chamber where we emulated conditions of high temperature storage conditions. Capacitors were placed in a controlled chamber whose temperature was maintained above their rated specification (IEC-60068-1, 1988). Pristine capacitors rated at $2200 \mu \mathrm{F}, 10 \mathrm{~V}$ and maximum rated storage temperature of $85^{\circ} \mathrm{C}$, from the same manufacturing lot were used for the experiment. The chamber temperature was gradually increased in steps of $25^{\circ} \mathrm{C}$ till the predetermined temperature limit was reached. The capacitors were allowed to settle at a set temperature for $15 \mathrm{~min}$ and then the next step increase was applied and was continued till the required temperature was attained. The above procedure was followed to decrease any possibility of shocks due to sudden change in the temperature variations.

Since the capacitors are rated at $85^{\circ} \mathrm{C}$ for storage conditions, for this experiment all the capacitors were subjected to a constant temperature of $105^{\circ} \mathrm{C}$ and humidity factor of $3.4 \%$, to observe degradation in the devices. At the end of specific time interval the temperature was lowered in steps of $25^{\circ} \mathrm{C}$ till the required room temperature was reached. Before measurements were made on the capacitors were kept at room temperature for $30 \mathrm{~min}$ to stabilize the temperature of the capacitor capsule. The $E S R$ value is the real impedance measured through the terminal software of the instrument. Similarly the capacitance value is computed from the imaginary impedance using Electrochemical Impedance Spectroscopy (EIS). An SP-150 Biologic impedance measurement instrument was used making the measurements (Biologic, 2010).

\subsection{Degradation Hypothesis and Results}

Figure 10 shows the Nyquist plots for a capacitor characterized under different stages of degradation during accelerated thermal aging. As the capacitors degrade, we observe a considerable tilt away from the axis which indicates decrease in the capacitance. In contrast the point of origin has a smaller deflection away from the axis indicating that there is a considerably smaller change in the measured $E S R$ value. Similarly the plots in Figure 11 shows the Bode plots for same capacitors when characterized at different time intervals in the aging cycle. From the magnitude plot we can observe the change in total impedance $(Z)$ of the capacitor with the frequency sweep as the device degrades. As the capacitors degrade the magnitude plot shifts upwards indicating an increase in the total impedance of the capacitance, which in this case, is the increase in $C$ and $E S R$ values. From the phase plot, it is seen that as the device degrades there is shift in phase indicating change in capacitance, which can also be observed clearly from the Nyquist plot in Figure 10.

In the thermal overstress experiments, the capacitors were characterized periodically upto 3400 hours of operation. It was observed that the average capacitance value decreased by more than $8-9 \%$ while decrease in the $E S R$ value was observed around $20-22 \%$. Therefore, the capacitance value came closer to its unhealthy value ( $\geq 10 \%$ degradation) whereas the $E S R$ value was well within specifications. Since the capacitance degradation is clearly observed and is more significant, the $C$ parameter was selected as a precursor to failure parameter to estimate the current health condition of the device.

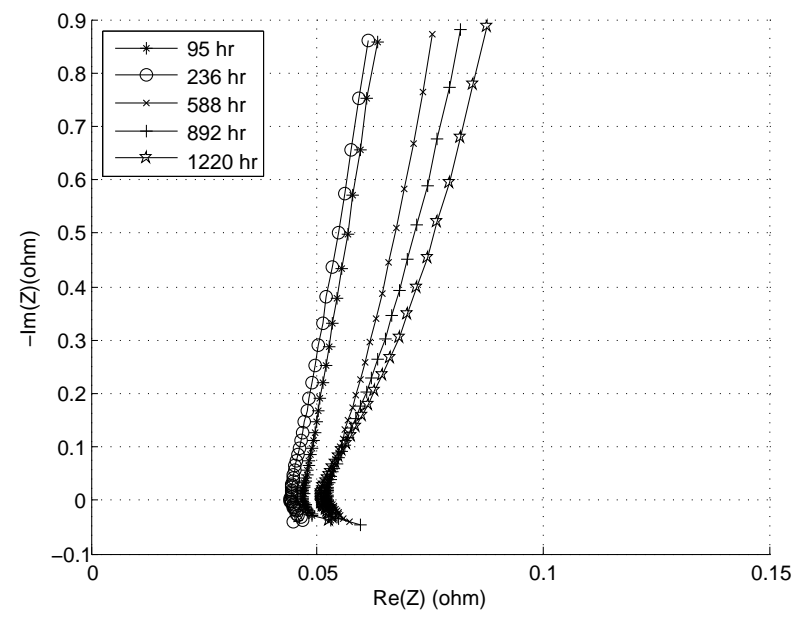

Figure 10. Nyquist Plot of a typical capacitor under Thermal Stress

Decrease in $C$ is plotted as a function of aging time. The gradual decrease in the capacitance can be related to the physics of failure model derived earlier. As the temperature increases, the electrolyte soaked in the capacitors evaporates. From the thermal model discussed, decrease in the capacitance is directly linked to the decrease in the effective oxide area $\left(A_{s}\right)$ of the capacitor. This decrease in the oxide area is in turn directly related to the rate of evaporation (Rodner et al., 2002) of the electrolyte given by Eq.( 13)

Figure 12 shows the plot for decrease in capacitance values for all the 15 capacitors (labeled capacitors \#1 through \#15) in the thermal overstress experiment. Till about 2400 hours of storage we observe a linear decrease in capacitance. This could be related to the electrolyte evaporation at a constant rate because the capacitor was stored at a fixed high temperature. As we continued the thermal aging cycle beyond this time period, from around 2450 hours onwards we observed a higher order effect that resulted in non-linear degradation. This non-linear phenomenon is hypothesized to be related to degradation in the oxide layer of the capacitor. In the early stages of aging, the linear degradation phenomena linked to 

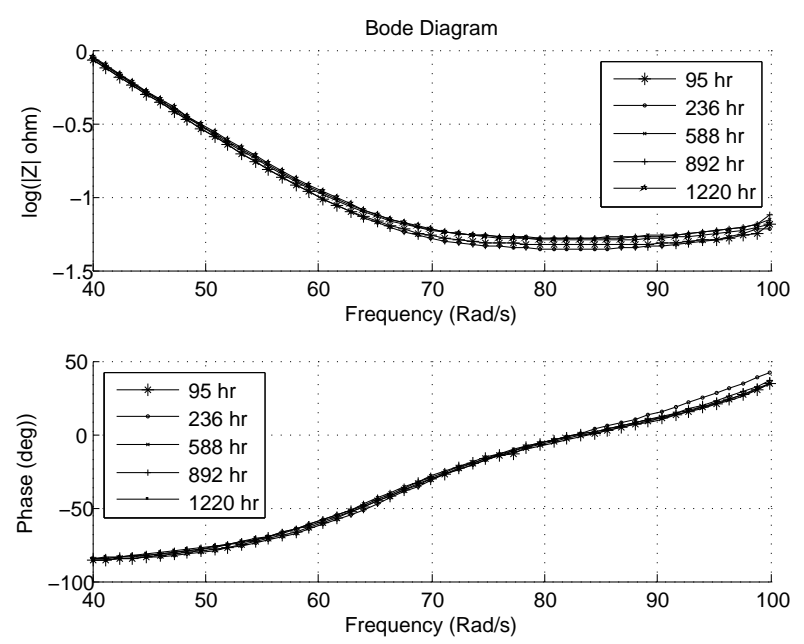

Figure 11. Bode Plot of a typical capacitor under Thermal Stress

electrolyte evaporation dominates, where as the non-linear degradation phenomenon due to oxide layer becomes dominant in the later part of the aging cycle. Currently in this work we have studied only the linear degradation due to electrolyte evaporation.

The relationship between capacitance and electrolyte volume is given by model $\mathcal{D}_{1}$ in Eq. (14), can be rewritten to express the change in electrolyte volume, $V_{e}$ over time given by :

$$
\mathcal{D}_{3}: V_{e}(t)=V_{e 0}-C(t)\left(\frac{j_{e o} t w_{e} d_{C}}{2 \epsilon_{R} \epsilon_{0}}\right)
$$

IN this model since the capacitors were subjected to a constant temperature the ethyl glycol evaporation rate $j_{e o}$ was considered to be constant (Rusdi et al., 2005).

\section{Model VAlidation and Discussion}

The time dependent model, $\mathcal{D}_{3}$ derived for estimating the electrolyte volume, $V_{e}$ implemented as a second order polynomial model for estimating the decrease in electrolyte volume over time. In this section we will discuss the parameter estimation for this model and study how well the developed degradation model, $\mathcal{D}_{1}$ behaves based on the estimated parameters.

\subsection{Parameter Estimation Approach}

Decrease in parameter, $C$ is used as a precursor of failure, from the experimental data and literature studies (IEC-600681, 1988; Rusdi et al., 2005). Based on the experiments, capacitance parameter values are computed by characterizing the capacitors at regular time intervals as shown in Figure 12. From the degradation model, $\mathcal{D}_{1}$ given a certain type of capacitor all the values in Eq. (14) can be computed except the dispersion in electrolyte volume, $V_{e}$, which is computed based on the available data and used degradation model, $\mathcal{D}_{1}$. Initial electrolyte volume $V_{e 0}$ at pristine conditions is approximately computed from the structural details of the capacitor as discussed in section (5.). From the experimental data at $105^{\circ} \mathrm{C}$ the estimated volume computed decreases almost linearly through the initial phase of degradation. Hence in this work we propose a linear model, which relates aging time to the decrease in loss of electrolyte volume. The loss in electrolyte is linked to decrease in capacitance through Eq. (14) and has the following form,

$$
\left(V_{e}\right) t_{k}=\hat{\theta_{1}}-\hat{\theta_{2}} t_{k}+\hat{\theta_{3}} t_{k}^{2}
$$

where $\hat{\theta_{1}}, \hat{\theta_{2}}$ and $\hat{\theta_{3}}$ are model constants for decrease in electrolyte volume $V_{e}$, estimated from the experimental data of accelerated thermal aging experiments. In order to estimate the model parameters, 14 capacitors out of the 15 used for the experiment, are used for estimation, and the remaining capacitor is used to validate the model against experimental data. This Leave-one-out cross-validation, methodology is implemented for validating the model against the computed capacitance degradation data. A nonlinear least-squares regression algorithm is used to estimate the model parameters.

\subsection{Parameter Estimation Results}

The goal of leave-one-out cross-validation is to estimate the expected level of fit of the derived physics-based model as discussed in Eq. (14). The errors from the results of the predicted model and actual data are the summarized in Tables (2) and (3), and compared using the mean $(\bar{X})$, median absolute deviation $(\tilde{X})$, root mean squared error (RMSE), root mean squared percentage error (RMSPE), standard deviation (SD), and confidence interval (CI) to study the overall predicted model behavior.

To demonstrate the approach we selected capacitors \#4 and \#5 from the set of 15 capacitors to show the detailed plots for volume and capacitance estimation. In Figure 13 based on the measurement data from capacitors \#1 - \#3 and \#5 through $\# 15$, volume parameters, $\hat{\theta_{1}}, \hat{\theta_{2}}$ and $\hat{\theta_{3}}$ were estimated. Degradation model, $\mathcal{D}_{3}$ was validated against the computed change in volume of capacitor $\# 4$, and model, $\mathcal{D}_{1}$ was validated for decrease in capacitance. Similarly for capacitor \#5 parameters were estimated based on data from capacitors \#1 - \#4 and \#6 through \#15. The estimated and computed values degradation in volume and capacitance respectively for capacitor \#5 are shown in plots of Figure 14.

It can be observed from the residuals of Figure 16 that the estimation error increases with time. This is to be expected since capacitance decrease is mostly linear till $2500 \mathrm{hrs}$, and then becomes non-linear, causing the residuals to increase. This indicates that there is a secondary phenomenon occur- 


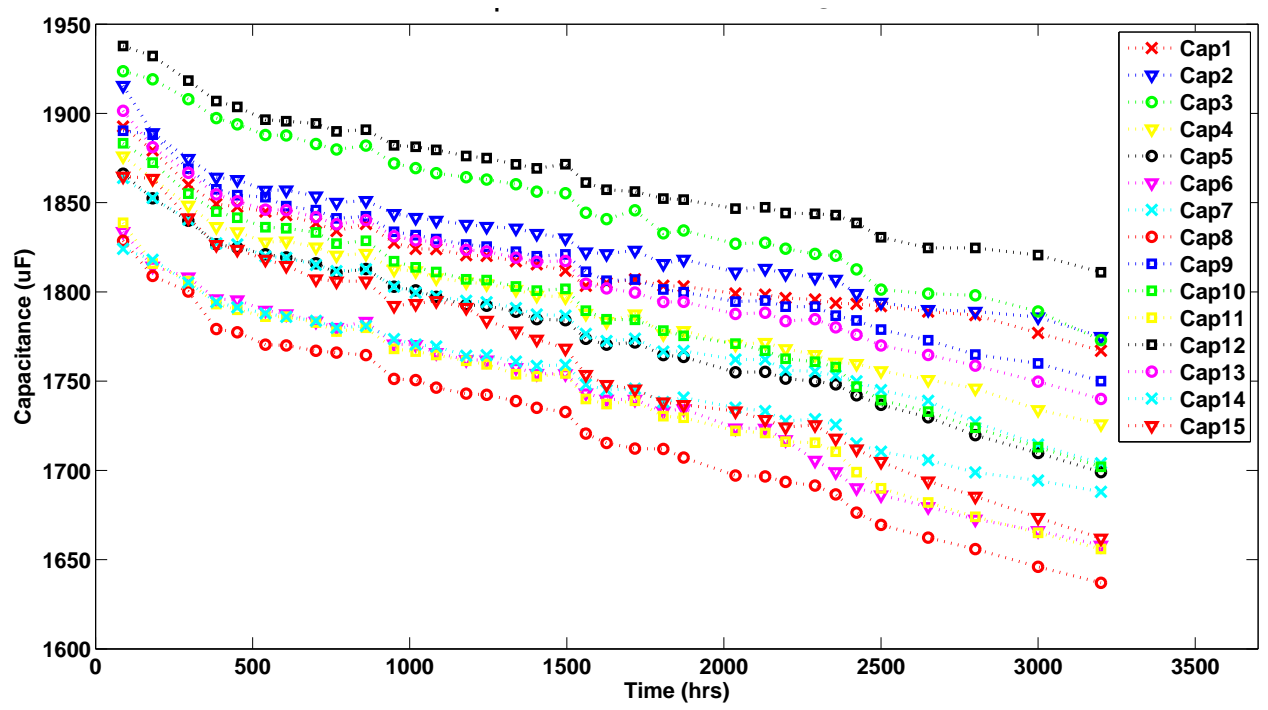

Figure 12. Thermal Overstress at $105 \mathrm{deg} \mathrm{C}-2200 \mathrm{uF}$
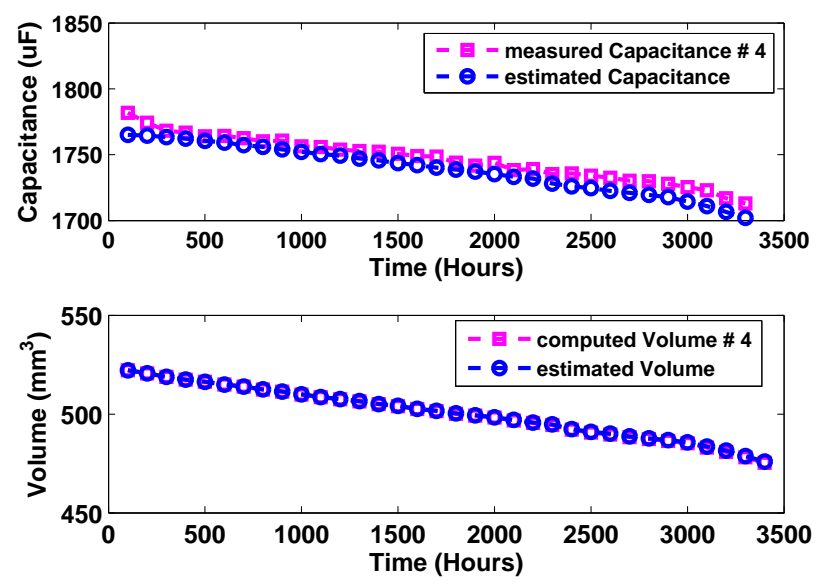

Figure 13. Volume and Capacitance Estimation (Cap \# 4)
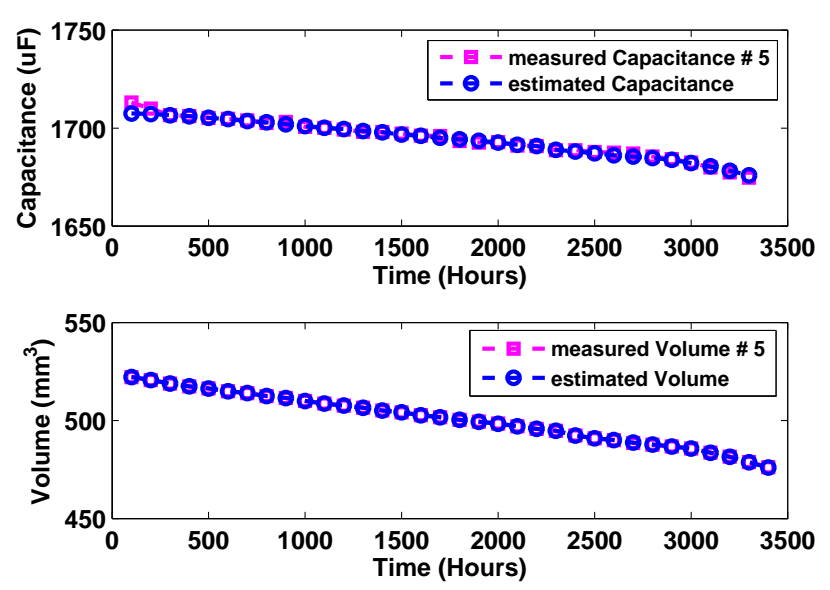

Figure 14. Volume and Capacitance Estimation ( Cap \# 5) ring in addition to the linear decrease due to loss in electrolyte volume, $V_{e}$. This is the oxide layer breakdown phenomenon which simultaneously degrades the capacitor and is exponential in nature. The phenomenon kicks-in prominently in the later stages of aging as seen from the data.

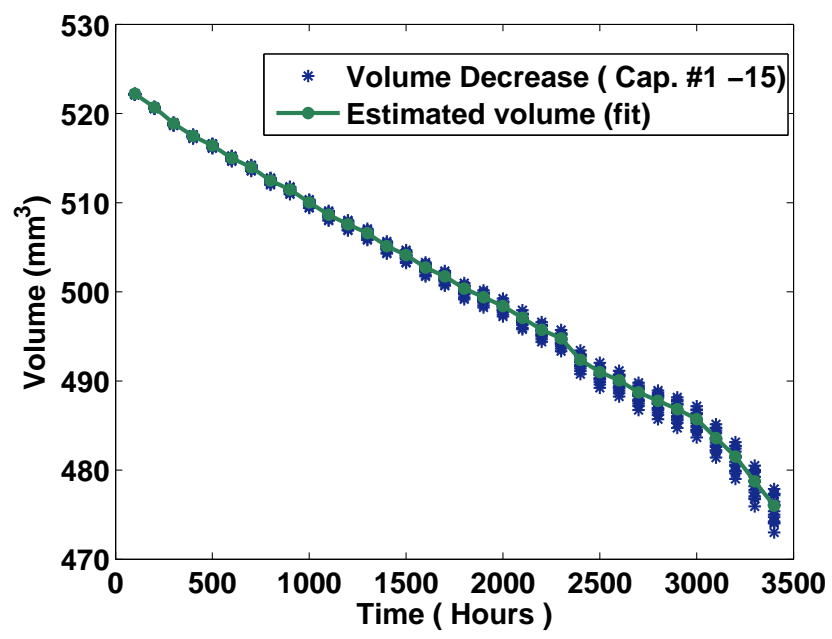

Figure 15. Estimation results for the degradation model

Table (2) summarizes the estimated parameters results for degradation in electrolyte volume in all the 15 capacitors under test are discussed in table (2). Root mean squared error (RMSE) is calculated for the difference between the computed electrolyte volume, $V_{e}$ and predicted electrolyte volume, $\hat{V}_{e}$ by degradation model, $\mathcal{D}_{3}$ while root mean square percentage error, RMSPE is computed from the average percentage error at different aging times for each capacitor.

The overall model parameters for the set of capacitors is sum- 


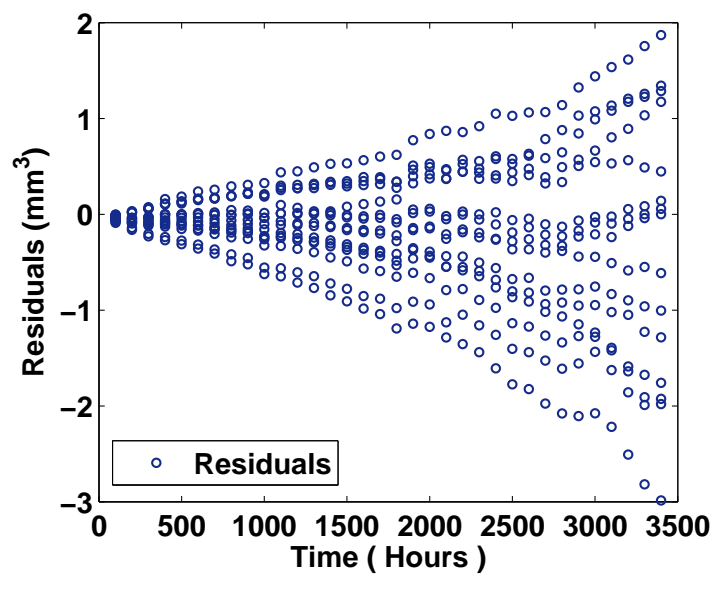

Figure 16. Electrolyte volume $\left(V_{e}\right)$ estimation residuals

marized in Table (3). $\bar{X}$ and $\tilde{X}$ give the mean and median values for $\hat{\theta_{1}}, \hat{\theta_{2}}$ and $\hat{\theta_{3}}$ respectively along with RMSE and RMSPE error parameters for each capacitor. Standard deviation and confidence interval for the overall model are also summarized in the table.

The parameters in Table (3) were used in degradation model, $\mathcal{D}_{1}$ to estimate the $(C)$ values. Figure 17 summarizes the plot showing residuals for difference between measured capacitance, $(C)$ and estimated capacitance, $(\hat{C})$ from the model, $\mathcal{D}_{1}$ for all the 15 capacitors. From the residuals it is observed that initially the residuals are high, as the capacitance calculated from the initial average estimated electrolyte volume value is higher. In the later stages of the aging cycle we the residual error increases due to the phenomenon of oxide breakdown discussed earlier in the section.

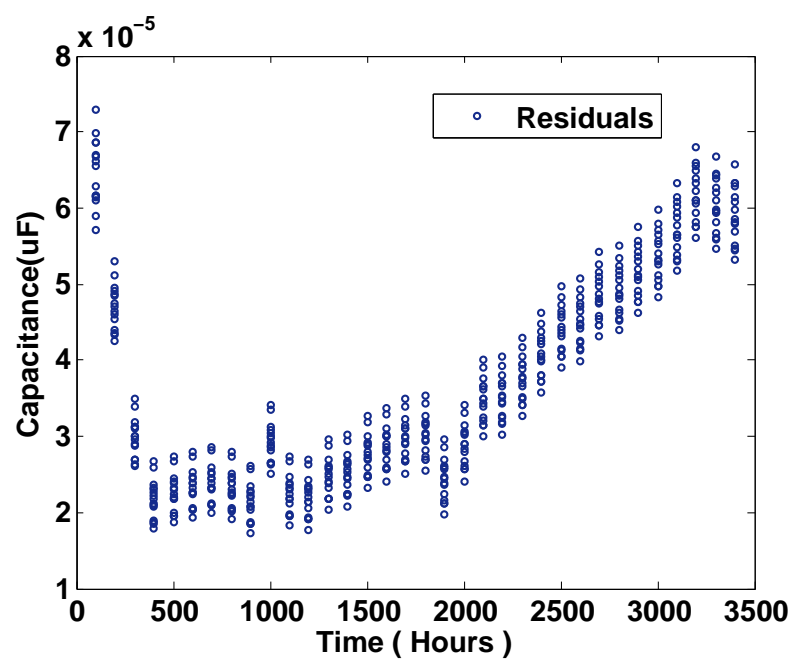

Figure 17. Capacitance $(C)$ measured and estimation residuals

\section{Discussion AND Conclusion}

This paper presents a first principles based degradation electrolytic capacitor model and a parameter estimation algorithm to validate the derived model, based on the experimental data. The majors contributions of the work presented in this paper are:

1. Identifying the key degradation mechanisms and failure modes based on operating conditions (fishbone diagram Figure 6).

2. Identification of the lumped-parameter model, $\mathcal{M}_{2}$ and $\mathcal{M}_{3}$, based on the equivalent electrical circuit of a real capacitor as a viable reduced-order model for prognostics-algorithm development;

3. Developing the thermal capacitance experiments based on the failure modes observed and collecting and analyzing data at regular intervals.

4. Identification of $C$ as a failure precursor in the lumped parameter model, $\mathcal{M}_{2}$ as shown in Figure 8;

5. Estimating the electrolyte volume from structural model of the capacitor to be implemented in the first principles degradation model, $\mathcal{D}_{3}$;

6. Development of the first principles degradation model based on accelerated life test aging data which includes decrease in capacitance as a function of time and evaporation rate linked to temperature conditions;

7. Implementation of parameter estimation algorithm to cross validate the derived first principles degradation model, $\mathcal{D}_{1}$.

The physics-based capacitance degradation model, $\mathcal{D}_{1}$ gives an indication of how a specific device degrades based on its geometric structure and operating conditions. The derived model can be updated and developed at a more finer granularity to be implemented for detailed prognostic implementation. The results presented here are based on accelerated aging experimental data and on the accelerated life timescale. This extends our previous work where we derived empirical degradation models from accelerated aging data.

The performance of the derived physics-based capacitance degradation model, $\mathcal{D}_{1}$ was successful based on the quality of the model fit to the experimental data and cross validation performance based on the parameter estimations done. A derived second order parameterized volume degradation model provided good results.

Our future work will focus on exploring a more detailed implementation of the physics-based degradation model using a Bayesian approach which can then be used for making more accurate degradation and failure predictions. The other focus point will be on using the physics-based degradation models to validate the capacitor data under different thermal conditions and also different capacitor structures. This will greatly 


\begin{tabular}{|l|l|l|l|l|l|}
\hline Case & $\hat{\theta_{1}}\left(\mathrm{~mm}^{3}\right)$ & $\hat{\theta_{2}}\left(\mathrm{~mm}^{2} / t\right)$ & $\hat{\theta_{3}}\left(\mathrm{~mm} / \mathrm{t}^{2}\right)$ & RMSE & RMSPE \\
\hline \hline 1 & 523.6123 & 0.01613 & $3.7100 * 10^{-7}$ & 26.1851 & 0.8577 \\
\hline 2 & 523.6122 & 0.01613 & $3.7099 * 10^{-7}$ & 26.1741 & 0.8573 \\
\hline 3 & 523.6159 & 0.01614 & $3.9403 * 10^{-7}$ & 26.1602 & 0.8568 \\
\hline 4 & 523.6109 & 0.01609 & $3.8072 * 10^{-7}$ & 26.2178 & 0.8587 \\
\hline 5 & 523.6128 & 0.01614 & $3.8428 * 10^{-7}$ & 26.2370 & 0.8594 \\
\hline 6 & 523.6100 & 0.01613 & $3.7867 * 10^{-7}$ & 26.2795 & 0.8608 \\
\hline 7 & 523.6081 & 0.01614 & $3.7269 * 10^{-7}$ & 26.2317 & 0.8592 \\
\hline 8 & 523.6089 & 0.01613 & $3.7988 * 10^{-7}$ & 26.3005 & 0.8614 \\
\hline 9 & 523.6111 & 0.01616 & $3.7447 * 10^{-7}$ & 26.1931 & 0.8579 \\
\hline 10 & 523.6122 & 0.01613 & $3.8470 * 10^{-7}$ & 26.2277 & 0.8591 \\
\hline 11 & 523.6076 & 0.01611 & $3.7350 * 10^{-7}$ & 26.2786 & 0.8607 \\
\hline 12 & 523.6065 & 0.01614 & $3.7313 * 10^{-7}$ & 26.1356 & 0.8560 \\
\hline 13 & 523.6147 & 0.01609 & $3.8906 * 10^{-7}$ & 26.2006 & 0.8592 \\
\hline 14 & 523.6120 & 0.01612 & $3.8276 * 10^{-7}$ & 26.2608 & 0.8601 \\
\hline 15 & 523.6113 & 0.01616 & $3.8317 * 10^{-7}$ & 26.2658 & 0.8603 \\
\hline
\end{tabular}

Table 2. Parameter Estimation for Degradation Model, $\mathcal{D}_{3}$

\begin{tabular}{|l|l|l|l|l|}
\hline Parameter & $\bar{X}$ & $\tilde{X}$ & S.D & C.I \\
\hline \hline$\hat{\theta_{1}}\left(\mathrm{~mm}^{3}\right)$ & 523.6112 & 523.6113 & 0.0026 & {$[523.6098,523.6127]$} \\
\hline$\hat{\theta_{2}}\left(\mathrm{~mm}^{2} / t\right)$ & 0.0161 & 0.0161 & $1.8748 * 10^{-5}$ & {$[0.01614,0.01611]$} \\
\hline$\hat{\theta_{3}}\left(\mathrm{~mm} / \mathrm{t}^{2}\right)$ & $3.8077 * 10^{-7}$ & $3.8072 * 10^{-7}$ & $6.9373 * 10^{-9}$ & {$\left[0.3769 * 10^{-6}, 0.3846^{*} 10^{-6}\right]$} \\
\hline RMSE & 26.2232 & 26.2277 & 0.0483 & {$[26.1965,26.2500]$} \\
\hline RMSPE & 0.8589 & 0.8591 & 0.0016 & {$[0.8580,0.8598]$} \\
\hline
\end{tabular}

Table 3. Error Summary for Degradation Model, $\mathcal{D}_{3}$

enhance the quality and effectiveness of the degradation models in prognostics, where the operating and environmental conditions along with the structural conditions are also accounted for towards degradation dynamics.

\section{NOMENCLATURE}

$\epsilon_{R} \quad$ relative dielectric constant

$\epsilon_{O} \quad$ permitivity of free space

$t_{o} \quad$ oxide thickness

$\mathrm{V}$ dispersion volume at time $t$

$V_{O} \quad$ initial electrolyte volume

$j_{e o} \quad$ evaporation rate $\left(\mathrm{hr}^{-1} \mathrm{~cm}^{-2}\right)$

$t \quad$ time in minutes

$\rho_{E} \quad$ electrolyte resistivity

$P_{E} \quad$ correlation factor related to electrolyte spacer porosity and average liquid pathway.

$r_{c} \quad$ radius of capacitor capsule

$h_{c} \quad$ height of capacitor capsule

$V_{\text {paper }} \quad$ volume of paper.

$A_{s} \quad$ effective oxide surface area ( $\left.\mathrm{L} \times \mathrm{H}\right)$

$w_{e} \quad$ volume of ethyl glycol molecule

$V_{c} \quad$ total capacitor capsule volume

$d_{A} \quad$ thickness of anode strip,

$d_{C} \quad$ thickness of cathode strip

$d_{s} \quad$ thickness of spacer strip

$\mathcal{M}_{1} \quad$ electrical lumped parameter model

$\mathcal{M}_{2} \quad$ simplified lumped parameter model

$\mathcal{M}_{3}$ basic lumped parameter model

$\mathcal{D}_{1} \quad$ Capacitance degradation model

$\mathcal{D}_{2} \quad$ ESR degradation model

$\mathcal{D}_{3} \quad$ Volume degradation model 


\section{REFERENCES}

Albella, J. M., Gomez-Aleixandre, C., \& Martinez-Duart, J. M. (1984). Dielectric characteristics of miniature aluminium electrolytic capacitors under stressed voltage conditions. Journal of Applied Electrochemistry, Volume 14, Number 1, 9 - 14.

Alwitt, R. S., \& Parler, S. G. (1995). Heat generation during pulse operation of prototype aluminum electrolytic capacitors. Journal of Applied Electrochemistry, Volume 25, Number 6, 533 - 542.

Balaban, E., Saxena, A., Narasimhan, S., Roychoudhury, I., Goebel, K., \& Koopmans, M. (2010). Airborne electro-mechanical actuator test stand for development of prognostic health management systems. Proceedings of Annual Conference of the PHM Society 2010, October 10-16, Portland, OR.

Bengt, A. (1995). Electrolytic capacitors theory and applications. RIFA Electrolytic Capacitors.

Biologic. (2010). Application note 14-zfit and equivalent electrical circuits [Computer software manual].

Brettle, J., \& Jackson, N. F. (1977). Failure mechanisms in solid electrolytic capacitors. Electrocomponent Science and Technology, 3, 233 - 246.

Buiatti, G., Martin-Ramos, J., Garcia, C., Amaral, A., \& Cardoso, A. (2010). An online and noninvasive technique for the condition monitoring of capacitors in boost converters. IEEE Transactions on Instrumentation and Measurement, 59, 2134 - 2143.

Campbell, D. S. (1971, January). Electrolytic capacitors. The Radio and Electronic Engineer, 41(1), 5 - 16.

Celaya, J., Kulkarni, C., Biswas, G., \& Goebel, K. (2011). A model-based prognostics methodology for electrolytic capacitors based on electrical overstress accelerated aging. Proceedings of Annual Conference of the PHM Society, September 25-29, Montreal, Canada.

Celaya, J., Saxena, A., Wysocki, P., Saha, S., \& Goebel, K. (2010). Towards prognostics of power mosfets: Accelerated aging and precursors of failure. Proceedings of Annual Conference of the PHM Society, October 1016, Portland, OR.

Celaya, J., Wysocki, P., Vashchenko, V., Saha, S., \& Goebel, K. (2010, September). Accelerated aging system for prognostics of power semiconductor devices. Proceedings of IEEE Autotestcon, 1-6.

Chen. (2004). Ripple current confusion [Computer software manual].

Daigle, M., \& Goebel, K. (2011). A model-based prognostics approach applied to pneumatic valves. International Journal of the PHM Society, Vol. 2(8), 1-16.

Ferrell, B. L. (1999). JSF prognostics and health management. IEEE Aerospace Conference.

FIDESGroup. (2004). Reliability methodology for electronic systems. FIDES Guide issue A.
Fife, J. (2006, August). Wet electrolytic capacitors (Patent No: 7,099 No. 1). Myrtle Beach, SC: AVX Corporation.

Gasperi, M. L. (1996, October). Life prediction model for aluminum electrolytic capacitors. 31st Annual Meeting of the IEEE-IAS, 4(1), 1347 - 1351.

Gomez-Aleixandre, C., Albella, J. M., \& Martinez-Duart, J. M. (1984). Gas evolution in aluminum electrolytic capacitors. Journal of Electrochem. Society, Volume 131, Issue 3, 612 - 614.

Goodman, D., Hofmeister, J., \& Judkins, J. (2007). Electronic prognostics for switched mode power supplies. Microelectronics Reliability, 47(12), 1902 - 1906.

Gu, J., Azarian, M. H., \& Pecht, M. G. (2008). Failure prognostics of multilayer ceramic capacitors in temperature-humidity-bias conditions. International Conference on Prognostics and Health Management.

Gu, J., \& Pecht, M. (2008). Prognostics and health management using physics-of-failure. 54th Annual Reliability and Maintainability Symposium (RAMS).

Hansen, P., \& McCluskey, P. (2007). Failure models in power device interconnects. European Conference on Power Electronics and Applications, 1 - 9.

IEC-60068-1. (1988). Environmental testing, part 1: General and guidance. IEC Standards.

Ikonopisov, S. (1977). Theory of electrical breakdown during formation of barrier anodic films. Electrochimica Acta, , Volume 22, Issue 10, 1077 - 1082.

Imam, A. M. (2007). Condition monitoring of electrolytic capacitors for power electronics applications. Unpublished doctoral dissertation, Georgia Institute of Technology.

Judkins, J. B., Hofmeister, J., \& Vohnout, S. (2007). A prognostic sensor for voltage regulated switch-mode power supplies. IEEE Aerospace Conference, 1, 1 - 8.

Kulkarni, C., Biswas, G., \& Koutsoukos, X. (2009). A prognosis case study for electrolytic capacitor degradation in dc-dc converters. Proceedings of Annual Conference of the PHM Society, San Diego, CA.

Kulkarni, C., Biswas, G., Koutsoukos, X., Goebel, K., \& Celaya, J. (2010, September). Diagnostic/prognostic experiments for capacitor degradation and health monitoring in dc-dc converters. SMASIS 2010.

Kulkarni, C., Celaya, J., Biswas, G., \& Goebel, K. (2011). Prognostic modeling and experimental techniques for electrolytic capacitor health monitoring. The 8th International Workshop on Structural Health Monitoring 2011 (IWSHM) , September 13-15, Stanford University, Stanford, $C A$.

Kulkarni, C., Celaya, J., Goebel, K., \& Biswas, G. (2012, July). Physics based electrolytic capacitor degradation models for prognostic studies under thermal overstress. Proceedings of First European Conference of the Prognostics and Health Management Society. 
Leonard, C., \& Pecht, M. G. (1991). Improved techniques for cost effective electronics. Proceedings of Reliability Maintainability Symposium, 174 - 182.

Luo, J., Namburu, M., Pattipati, K., \& et'al. (2003, Sep). Model-based prognostic techniques. AUTOTESTCON 2003. IEEE Systems Readiness Technology Conference. Proceedings, 330 - 340.

Morley, A. R., \& Campbell, D. S. (1973, July). Electrolytic capacitors: Their fabrication and the interpretation of their operational behaviour. The Radio and Electronic Engineer, 43(7), 421 - 429.

Nasser, L., \& Curtin, M. (2006, March). Electronics reliability prognosis through material modeling and simulation. Proceedings of IEEE Aerospace Conference.

Nie, L., Azarian, M., Keimasi, M., \& Pecht, M. (2007). Prognostics of ceramic capacitor temperature humidity bias reliability using mahalanobis distance. Circuit World, 33(3), $21-28$.

Orsagh, R., \& et'al. (2006, March). Prognostic health management for avionics system power supplies. Aerospace Conference, 2006 IEEE, 1 - 7.

Pecht, M., Dube, M., Natishan, M., \& Knowles, I. (2001). An evaluation of built-in test. IEEE Transactions on Aerospace and Electronic Systems, 37, 266 - 272.

Rodner, S. C., Wedin, P., \& Bergstram, L. (2002). Effect of electrolyte and evaporation rate on the structural features of dried silica monolayer films. Langmuir, 18(24), 9327 - 9333.

Roederstein, V. (2007). Aluminum capacitors - general information. Document - 25001 January 2007.

Rusdi, M., Moroi, Y., Nakahara, H., \& Shibata, O. (2005). Evaporation from water ethylene glycol liquid mixture. Langmuir - American Chemical Society, 21 (16), 7308 $-7310$.

Saha, B., \& Goebel, K. (2011). Model adaptation for prognostics in a particle filtering framework. International Journal of the PHM Society, Vol. 2(6), 1-12.

Tasca, D. M. (1981). Pulse power response and damage characterization of capacitors. Electrical Overstress-Electrostatic Discharge Symposium Proceedings, EOS/ESD Association, 3.

Upadhyayula, K., \& Dasgupta, A. (1998). Guidelines for physics-of-failure based accelerated stress testing. Annual Reliability and Maintainability Symposium, 345 357.

Vichare, N., \& Pecht, M. (2006). Prognostics and health management of electronics. IEEE Transactions on Components and Packaging Technologies, Vol 29, 222 - 229.

Vohnout, S., Kozak, M., Goodman, D., Harris, K., \& Judkins, J. (2008). Electronic prognostics system implementation on power actuator components. Aerospace Conference, 2008 IEEE, 1 - 11.
Watson, G. F. (1992). Mil reliabiliy: A new approach. IEEE Spectrum, August 1992, 46 - 49.

Wereszczak, A., Breder, K., \& Ferber, M. K. (1998). Failure probability prediction of dielectric ceramics in multilayer capacitors. Annual Meeting of the American Ceramic Society, Cincinnati, OH (United States).

Wit, H. D., \& Crevecoeur, C. (1974). The dielectric breakdown of anodic aluminum oxide. Physics Letters A, Volume 50, Issue 5, 365 - 366.

Chetan S. Kulkarni is a research engineer II with SGT Inc. at the Prognostics Center of Excellence, NASA Ames Research Center. He received the M.S. and Ph.D. from Vanderbilt University, Nashville, TN, in 2009 and 2013, respectively. He received the B.E. (Bachelor of Engineering) degree in Electronics and Electrical Engineering from University of Pune, India in 2002. The research work in this paper was done by Chetan when he was a Ph.D. candidate at Vanderbilt University.

Gautam Biswas received the Ph.D. degree in computer science from Michigan State University, East Lansing. He is a Professor of Computer Science and Computer Engineering in the Department of Electrical Engineering and Computer Science, Vanderbilt University, Nashville, TN.

José R. Celaya is a research scientist with SGT Inc. at the Prognostics Center of Excellence, NASA Ames Research Center. He received a Ph.D. degree in Decision Sciences and Engineering Systems in 2008, a M. E. degree in Operations Research and Statistics in 2008, a M. S. degree in Electrical Engineering in 2003, all from Rensselaer Polytechnic Institute, Troy New York; and a B. S. in Cybernetics Engineering in 2001 from CETYS University, México.

Kai Goebel received the degree of Diplom-Ingenieur from the Technische Universität München, Germany in 1990. He received the M.S. and Ph.D. from the University of California at Berkeley in 1993 and 1996, respectively. Dr. Goebel is the Deputy Area Lead for Discovery and System Health at NASA Ames Research Center where he also leads the Diagnostics and Prognostics groups in the Intelligent Systems division. In addition, he directs the Prognostics Center of Excellence and he is the technical lead for Prognostics and Decision Making of NASA's System-wide Safety and Assurance Technologies Program. He worked at General Electric's Corporate Research Center in Niskayuna, NY from 1997 to 2006 as a senior research scientist. He has carried out applied research in the areas of artificial intelligence, soft computing, and information fusion. His research interest lies in advancing these techniques for real time monitoring, diagnostics, and prognostics. He holds 17 patents and has published more than 250 papers in the area of systems health management. 